\title{
High serum gamma-glutamyl transpeptidase concentration associates with poor postoperative prognosis of patients with hepatitis B virus-associated intrahepatic cholangiocarcinoma
}

\author{
Bo Zhang ${ }^{1,2 \#}$, Shuang Liu ${ }^{3 \#}$, Binghai Zhou ${ }^{1,2 \#}$, Lei Guo ${ }^{1,2}$, Hui Li ${ }^{1,2}$, Jiuliang Yan ${ }^{1,2}$, Wentao Zhang ${ }^{1,2}$, \\ Mincheng Yu ${ }^{1,2}$, Zheng Chen ${ }^{1,2}$, Yongfeng $\mathrm{Xu}^{1,2}$, Yongsheng Xiao ${ }^{1,2}$, Qinghai Ye, \\ ${ }^{1}$ Department of Liver Surgery and Transplantation, Liver Cancer Institute and Zhongshan Hospital, Fudan University, Shanghai, China; ${ }^{2}$ Key \\ Laboratory of Carcinogenesis and Cancer Invasion (Fudan University), Ministry of Education, Fudan University, Shanghai, China; ${ }^{3}$ Department of \\ Neurosurgery, Zhongshan Hospital, Fudan University, Shanghai, China \\ Contributions: (I) Conception and design: Q Ye, Y Xiao, B Zhang, S Liu; (II) Administrative support: L Guo, H Li; (III) Provision of study materials or \\ patients: J Yan, W Zhang, M Yu, Z Chen, Y Xu; (IV) Collection and assembly of data: B Zhang, S Liu, B Zhou; (V) Data analysis and interpretation: \\ B Zhang, S Liu; B Zhou; (VI) Manuscript writing: All authors; (VII) Final approval of manuscript: All authors. \\ \#These authors contributed equally to this work. \\ Correspondence to: Qinghai Ye; Yongsheng Xiao. Department of Liver Surgery and Transplantation, Liver Cancer Institute and Zhongshan Hospital, \\ Fudan University, 180 Feng Lin Road, Shanghai 200032, China. Email: ye.qinghai@zs-hospital.sh.cn; Xiao.yongsheng@zs-hospital.sh.cn.
}

Background: Intrahepatic cholangiocarcinoma (ICC) caused by chronic hepatitis B virus (HBV) infection has become prominent. Prospectively stratifying postoperative risk factors is a challenging task.

Methods: We retrospectively assessed the relationship between serum gamma-glutamyl transpeptidase (GGT) concentration and postoperative outcomes in 107 subjects with HBV-associated ICC. Cox proportionate hazard models and subgroup analyses were used to test the hypothesis with adjustment for potential confounders.

Results: Serum GGT concentration was negatively correlated with postoperative outcomes. For a 1-standard deviation (per-SD) $(117 \mu / \mathrm{L})$ increase of serum GGT concentration, the relative risk (RR) for overall survival (OS) and time to recurrence (TTR) were 1.72 [95\% confidence interval (CI), 1.37 to 2.16] and 1.53 (95\% CI, 1.22 to 1.91), respectively. In addition, the RRs of middle and top tertiles of GGT for death were 1.81 (95\% CI, 0.98 to 3.32 ) and 3.56 (95\% CI, 1.97 to 6.42 ), respectively ( $\mathrm{P}$ for trend $<0.001$ ). Similarly, the RRs for recurrence of the corresponding tertiles were 1.70 (95\% CI, 0.93 to 3.10 ) and 3.27 ( $95 \%$ CI, 1.77 to 6.06 ), respectively ( $\mathrm{P}$ for trend $=0.002$ ). In our study, the negative correlation between serum GGT levels and OS did not differ significantly between groups stratified by age, sex, HBV DNA level, carbohydrate antigen 19-9 (CA19-9) level and liver resection type (all P for interaction >0.05); however, there was a significant interactive effect of serum GGT and adjuvant chemotherapy on OS ( $\mathrm{RR}=0.64 v s .1 .77$, $\mathrm{P}$ for interaction $=0.04$ ).

Conclusions: High serum GGT concentration is associated with an increased risk of postoperative death and tumor recurrence in patients with HBV-associated ICC. However, this relationship became less significant with the implementation of adjuvant chemotherapy.

Keywords: Gamma-glutamyl transpeptidase (GGT); chronic hepatitis B virus infection (chronic HBV infection); intrahepatic cholangiocarcinoma (ICC); prognosis

Submitted Feb 15, 2020. Accepted for publication Oct 10, 2020.

doi: 10.21037/atm-20-1616

View this article at: http://dx.doi.org/10.21037/atm-20-1616 


\section{Introduction}

Intrahepatic cholangiocarcinoma (ICC) is the second most common primary hepatic malignancy after hepatocellular carcinoma (HCC), representing 10-15\% of primary hepatic malignancies and its prevalence is constantly rising worldwide (1). Currently, surgical treatment is the only curative therapy for ICC, so if a patient has a potentially resectable tumor, it is the preferred treatment (2). Unfortunately, rates of recurrence or metastasis after radical resection are reported as high as 60-65\% (3). This may explain why 5 -year survival rate has not improved significantly in this decade (4).

Therefore, biomarkers that can indicate postoperative outcomes may have important clinical significance for guiding treatment, adjuvant therapy, and follow-up strategies.

Recently, several epidemiological studies have suggested the correlation between serum gamma-glutamyl transpeptidase (GGT) concentration and the prognosis of various types of cancers (5), including ICC (6-8). In this study, we focus on patients with hepatitis B virus (HBV)associated ICC because chronic HBV infection or HBVassociated cirrhosis have been reported by many studies to play an important role in the development of ICC, especially where $\mathrm{HBV}$ is endemic $(9,10)$. In addition, ICC caused by HBV infection differs from other etiologies markedly in tumor origin, tumor microenvironment, oncogenic signaling pathway, biological characteristics, and can lead to different postoperative outcomes $(8,9,11,12)$. Thus, it is meaningful to evaluate the correlation between GGT and this specific carcinoma.

To work out this knowledge gap, we retrospectively analyzed the relationship between serum GGT and postoperative outcomes in a cohort of patients with $\mathrm{HBV}$-associated ICC. Particularly, we conducted a series of sensitivity analyses to verify this hypothesis. To our knowledge, this is the first epidemiologic investigation that provides reliable observational evidence on the role of GGT in the postoperative prognosis of $\mathrm{HBV}$-associated ICC.

We present the following article in accordance with the STROBE reporting checklist (available at http://dx.doi. org/10.21037/atm-20-1616).

\section{Methods}

\section{Ethics approval and consent to participate}

This study retrospectively evaluated patient data from our electronic medical record database, and all patient identities were anonymized before analyses. The study protocol was approved by the Zhongshan Hospital Research Ethics Committee (Y2017-250) and individual consent for this retrospective analysis was waived. The entire study process complied with the ethical guidelines of the 1975 Declaration of Helsinki (as revised in Brazil in 2013).

\section{Study population}

We obtained the patients' data from electronic medical record database in the Department of Liver Surgery, Zhongshan Hospital. We defined HBV-associated ICC as seropositivity for hepatitis B surface antigen (HBsAg) or hepatitis $\mathrm{B}$ core antibody (HBcAb) while being diagnosed as ICC, because these two indicators are often used to indicate chronic hepatitis B infection (13). Adult patients who underwent surgery for $\mathrm{HBV}$-associated ICC from January 2006 to December 2010 and had complete follow-up data were identified. We enrolled participants who also met the following criteria: (I) without history of other malignancies, (II) no evidence of distant tumor metastasis, (III) no preoperative anticancer treatment, (IV) macroscopic curative resection with negative margins, (V) histopathologically confirmed ICC. Figure 1 shows complete exclusion process of this study. Finally, a total of 107 patients were included.

\section{Assessment covariates}

Based on previously published literatures regarding covariates affecting the prognosis of hepatobiliary tumors, we extracted patients' baseline characteristics including demographics, liver function-related indicators, and serum tumor markers. Additionally, we collected tumor-related parameters, such as tumor size, number, location, capsule, microvascular invasion, lymph node metastasis and histologic differentiation. Finally, information on resection type and postoperative adjuvant chemotherapy were also included.

Detailed data on patients' baseline characteristics were obtained at the time of admission. Serum creatinine, total bilirubin (TB), alanine aminotransferase (ALT), GGT and albumin were measured using automatic clinical analyzers (Beckman Coulter) at the core laboratory of the Zhongshan hospital. The serum HBV-DNA level was quantified by PCR (ABI 7300, Applied Biosystems, Foster City, CA, USA) with a quantification linear range from 


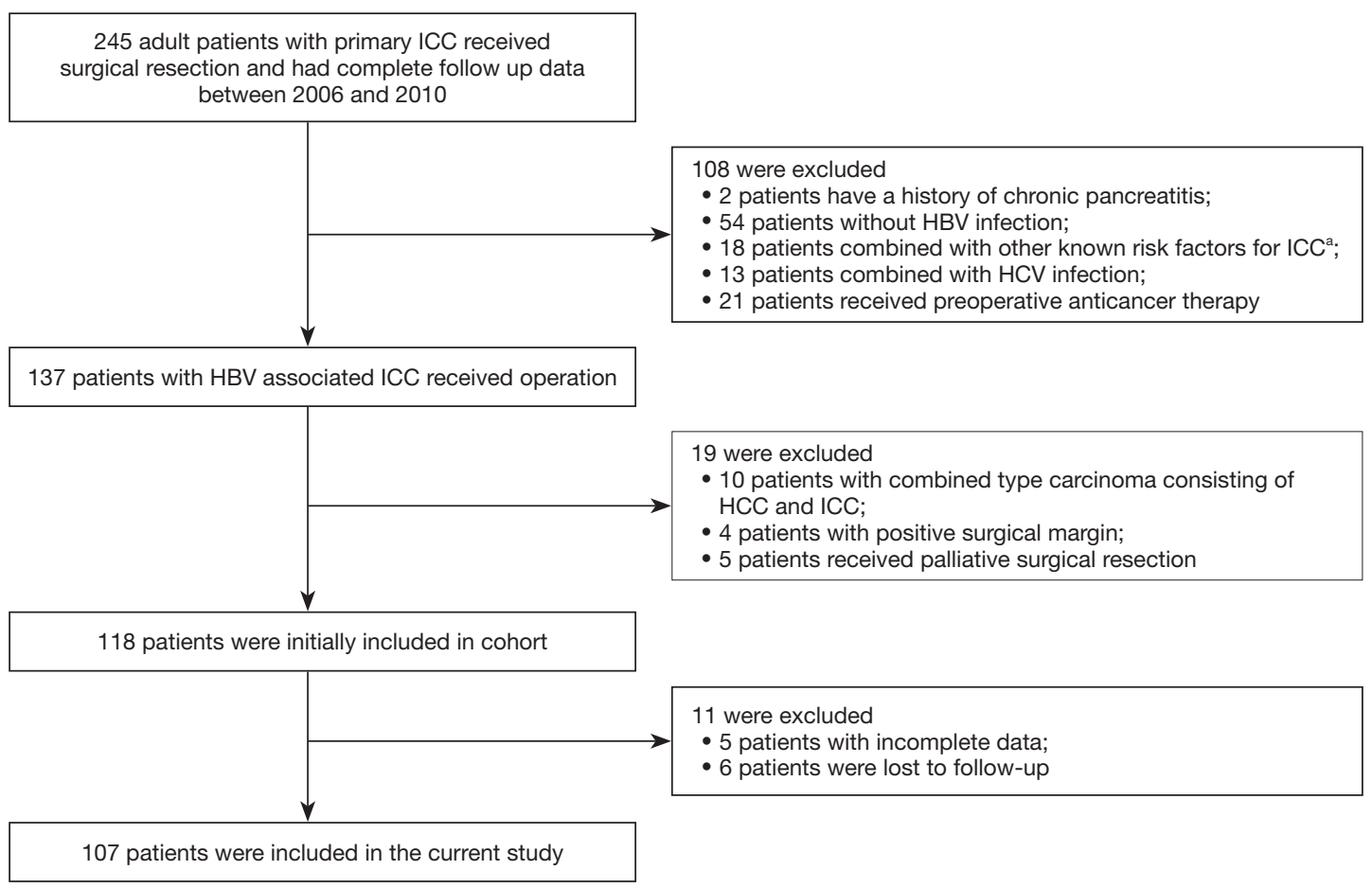

Figure 1 The study flow chart. ', these diseases are considered to be the known risk factors for ICC: liver fluke infection, primary sclerosing cholangitis and hepatolithiasis. ICC, intrahepatic cholangiocarcinoma; HCC, hepatocellular carcinoma; HBV, hepatitis B virus; HCV, hepatitis $\mathrm{C}$ virus.

1,000 to $2,000,000 \mathrm{IU} / \mathrm{mL}$. For patients with HBV DNA $>10^{3} \mathrm{IU} / \mathrm{mL}$, antiviral therapy was performed and reevaluated at follow up to maintain the viral replication at a low level. Briefly, patients with good general performance, adequate liver function reserve, technically resectable tumors and without distant metastasis were recommended for operation after discussion by the surgery team.

Tumor size, number, location and resection type were acquired from operation records. Tumor capsule, microvascular invasion, lymph node metastasis and histologic differentiation were evaluated using postoperative histopathological reports. The histopathological diagnosis of ICC was based on the 2000 version of the World Health Organization classification. Histologic grade was assessed according to the Edmondson-Steiner grading system based on the highest grade in the specimen. After the operation, some patients received adjuvant chemotherapy according to their wishes. This therapy mainly consists of intravenous administration of gemcitabine at a standard dose of $1,000 \mathrm{mg} / \mathrm{m}^{2}$ and cisplatin at a standard dose of $100 \mathrm{mg} / \mathrm{m}^{2}$, every 2 weeks (GEMOX) with possible dose adjustments. Since we only enrolled patients with radical resection, no study cases received adjuvant radiotherapy.

\section{Follow up and outcomes}

In current study, we reported perioperative complications, according to the Clavien-Dindo standardized classification system, which was recommended for retrospective studies in its current form (14). After discharge, all patients were regularly followed up in our hospital every 3 months for the first 2 years and every 6 months thereafter. The routine followup investigations included a detailed history and a complete physical examination. In addition, serum tumor markers, liver function tests, and abdominal ultrasound were also carried out. Contrast-enhanced computed tomography and/ or magnetic resonance imaging were performed once every 6 months or earlier when tumor recurrence or metastasis was suspected. The primary outcome was overall survival (OS) and the secondary outcome was time to recurrence (TTR). OS was defined as the time from the operation to confirmed death from all causes. TTR was calculated from surgery to the date when recurrence/metastasis was diagnosed. Data were summarized at the end of December 2015. 
Table 1 Patients' clinicopathological characteristics ${ }^{\#}$

\begin{tabular}{|c|c|c|}
\hline Variables & Value 1 & Value 2 \\
\hline Age, years & $51[46-61]$ & $53[11]$ \\
\hline $\mathrm{TB}(\mu \mathrm{mol} / \mathrm{L})$ & $12.5[10.2-16.6]$ & $14[6.5]$ \\
\hline Prothrombin time (s) & 11.6 [10.9-12.5] & $11.7[1.1]$ \\
\hline Serum albumin $(g / L)$ & $44.0[39.0-46.0]$ & $43[5]$ \\
\hline $\begin{array}{l}\text { Glutamic pyruvic } \\
\text { transaminase }(\mu / L)\end{array}$ & $34[23-50]$ & $47[47]$ \\
\hline GGT $(\mu / L)$ & 59 [32-120] & $102[117]$ \\
\hline Tumor size $(\mathrm{cm})$ & $5.8[4.0-7.5]$ & $6.1[2.6]$ \\
\hline Sex (male vs. female) & 72 vs. 35 & 67.3 vs. 32.7 \\
\hline $\begin{array}{l}\text { Cirrhosis (positive vs. } \\
\text { negative) }\end{array}$ & 14 vs. 93 & 13.1 vs. 86.9 \\
\hline $\begin{array}{l}\text { HBV DNA, copies } / \mathrm{mL}\left(\leq 10^{3}\right. \\
\left.\text { vs. }>10^{3}\right)\end{array}$ & 22 vs. 85 & 20.6 vs. 79.4 \\
\hline $\mathrm{CA} 19-9, \mu / \mathrm{mL}(\leq 37$ vs. $>37)$ & 52 vs. 55 & 48.6 vs. 51.4 \\
\hline AFP, ng/mL ( $\leq 20$ vs. $>20)$ & 82 vs. 25 & 76.6 vs. 23.4 \\
\hline $\begin{array}{l}\text { Tumor number (solidary vs. } \\
\text { multiple) }\end{array}$ & 100 vs. 7 & 93.5 vs. 6.5 \\
\hline $\begin{array}{l}\text { Tumor location (single lobe } \\
\text { vs. multiple lobes) }\end{array}$ & 91 vs. 16 & 85.0 vs. 14.5 \\
\hline $\begin{array}{l}\text { Liver resection (local vs. } \\
\text { anatomical) }\end{array}$ & 44 vs. 63 & 41.1 vs. 58.9 \\
\hline $\begin{array}{l}\text { Tumor capsule (negative vs. } \\
\text { positive) }\end{array}$ & 92 vs. 15 & 86.0 vs. 14.0 \\
\hline $\begin{array}{l}\text { Micro tumor thrombus (yes } \\
\text { vs. no) }\end{array}$ & 22 vs. 85 & 20.6 vs. 79.4 \\
\hline $\begin{array}{l}\text { Hilar lymph nodes (negative } \\
\text { vs. positive) }\end{array}$ & 97 vs. 10 & 90.7 vs. 9.35 \\
\hline $\begin{array}{l}\text { Histologic differentiation (well } \\
\text { vs. poor) }\end{array}$ & 70 vs. 37 & 65.4 vs. 34.6 \\
\hline $\begin{array}{l}\text { Adjuvant chemotherapy (yes } \\
\text { vs. no) }\end{array}$ & 36 vs. 71 & 33.6 vs. 66.4 \\
\hline \multicolumn{3}{|c|}{$\begin{array}{l}\text { ", value 1: continuous variables are shown as median [IQR], } \\
\text { categorical variables are shown as no.; value 2: continuous } \\
\text { variables are shown as mean [SD], categorical variables are } \\
\text { shown as \%. IQR, inter-quartile range; SD, standard deviation; } \\
\text { HBV, hepatitis B virus; TB, total bilirubin; GGT, gamma-glutamyl } \\
\text { transpeptidase; CA19-9, carbohydrate antigen 19-9; AFP, alpha- } \\
\text { fetoprotein. }\end{array}$} \\
\hline
\end{tabular}

\section{Statistics}

The relationship between serum GGT concentration and the risk of outcomes was evaluated with the use of serum GGT concentration as both a continuous variable
[1 standard deviation (per SD)] and a categorical variable (tertile). In addition, HBV DNA, carbohydrate antigen 19-9 (CA19-9) and alpha-fetoprotein (AFP) were categorized by clinical references to be calculated for relative risk (RR) and adjusted in the multivariate analysis.

We first described patients' baseline characteristics (Table 1). Then, we adopted univariate analysis (Table S1) and correlation analysis (Figure S1) to explore the potential confounders. Next, we adopted the Cox proportional hazards models adjusted for different confounders to estimate the independent relationship between serum GGT concentration and postoperative outcomes (Table 2). The "E-value", a new measurement related to evidence for causality in observational study, is calculated by online website (https:// www.evalue-calculator.com). The effects of baseline serum GGT concentration (tertile) on OS and TTR were also validated with the use of Kaplan-Meier curves (Figure 2). Subgroup analysis was used to examine the robustness of the primary results on the basis of pre-specified variables and compared the heterogeneity of results across subgroups using likelihood ratio test (Figure 3). Finally, we used chi-square test to compare the incidence of complications in different concentration of GGT groups (Table 3).

All data were double entered and then exported to tabdelimited text files. Analyses were conducted by $\mathrm{R}$ version 3.4.0 (http://www.R-project.org), using two-sided tests with a significance level of 0.05 .

\section{Results}

\section{Study characteristics}

As shown in Figure 1, a total of 107 patients are included in the present study and their characteristics were outlined in Table 1. The median age of the patients was 51 [inter-quartile range (IQR), 46-61] years. Of them, 72 (67.3\%) patients were men and $14(13.1 \%)$ patients were found to have cirrhosis. A majority of patients (79.4\%) had detectable serum HBV DNA ( $>10^{3}$ units $\left./ \mathrm{mL}\right)$ and then received antiviral therapy after admission. Micro tumor thrombus and hilar lymph node metastasis were detected in $22(20.6 \%)$ and $10(9.35 \%)$ patients, respectively. Overall, the median baseline serum GGT concentration of the patients was 59 (IQR, 32-120) $\mu / \mathrm{L}$ and the SD was $117 \mu / \mathrm{L}$.

\section{Multivariate analysis}

Multivariate analysis showed serum GGT concentration 
Table 2 Association of serum GGT concentration with OS and TTR

\begin{tabular}{|c|c|c|c|c|c|}
\hline Multivariate analysis & Per SD increase & Low tertile $(n=36)$ & Middle tertile $(n=35)$ & Top tertile $(n=36)$ & $\mathrm{P}$ trend \\
\hline Non-adjusted & $1.54(1.27,1.87)$ & 1.0 & $1.43(0.80,2.56)$ & $2.83(1.62,4.96)$ & 0.001 \\
\hline Model I ${ }^{a}$ & $1.57(1.28,1.92)$ & 1.0 & $1.75(0.96,3.20)$ & $3.24(1.81,5.81)$ & $<0.001$ \\
\hline Model II & $1.72(1.37,2.16)$ & 1.0 & $1.81(0.98,3.32)$ & $3.56(1.97,6.42)$ & $<0.001$ \\
\hline Non-adjusted & $1.53(1.26,1.86)$ & 1.0 & $1.53(0.87,2.68)$ & $3.10(1.79,5.37)$ & $<0.001$ \\
\hline Model III ${ }^{\mathrm{C}}$ & $1.51(1.22,1.87)$ & 1.0 & $1.68(0.93,3.05)$ & $3.23(1.78,5.86)$ & 0.001 \\
\hline Model IV ${ }^{d}$ & $1.53(1.22,1.91)$ & 1.0 & $1.70(0.93,3.10)$ & $3.27(1.77,6.06)$ & 0.002 \\
\hline
\end{tabular}

${ }^{a}$, these factors were adjusted: serum albumin, tumor location and histologic differentiation; ${ }^{b}$, these factors were adjusted: age, sex, prothrombin, serum albumin, tumor size, tumor location, micro thrombus invasion, histologic differentiation and adjuvant chemotherapy; ${ }^{c}$, these factors were adjusted: sex, serum albumin, tumor location, micro thrombus invasion and histologic differentiation; ${ }^{d}$, these factors were adjusted: age, sex, serum albumin, tumor size, tumor location, micro thrombus invasion, histologic differentiation and adjuvant chemotherapy. GGT, gamma-glutamyl transpeptidase; OS, overall survival; TTR, time to recurrence; SD, standard deviation.
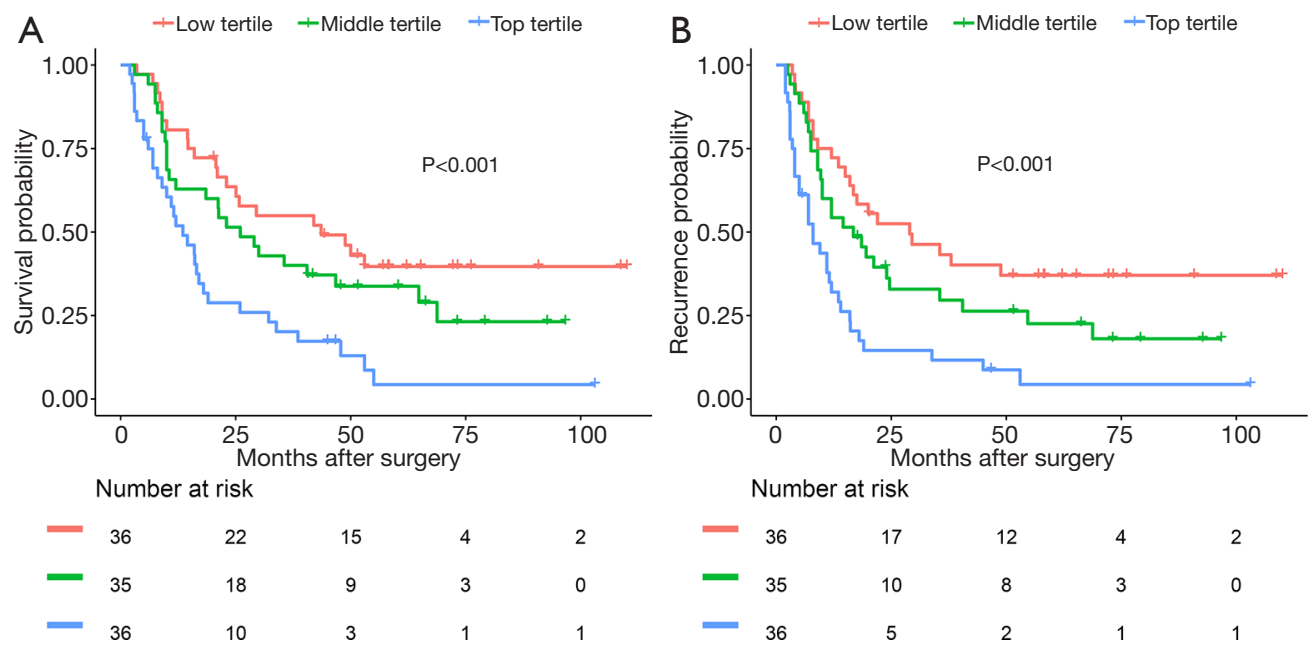

Figure 2 Kaplan-Meier survival curves of OS and TTR. (A) The proportion of survival over time was highest in low tertiles and lowest in top tertiles; (B) the proportion of tumor recurrence over time was highest in low tertiles and lowest in top tertiles. OS, overall survival; TTR, time to recurrence.

was independently correlated with OS and TTR whether it was defined as a continuous or a categorical variable (Table 2). For a per-SD $(117 \mu / \mathrm{L})$ increase in serum GGT concentration, the RRs for OS and TTR were 1.57 [95\% confidence interval (CI), 1.28 to 1.92 ] and 1.51 (95\% CI, 1.22 to 1.87 ), respectively, after the adjustment of potential confounding factors selected by their associations with the outcomes (Table S1) and serum GGT concentration (Figure S1). Even more confounding variables were adjusted progressively, serum GGT concentration remained significantly associated with $\mathrm{OS}(\mathrm{RR}=1.72,95 \% \mathrm{CI}, 1.37$ to 2.16 ) and TTR ( $\mathrm{RR}=1.53,95 \% \mathrm{CI}, 1.22$ to 1.91 ). In addition, we calculated the E-value for the observed association estimate (after adjustments for measured confounders) and $\mathrm{E}=2.83$ (95\% CI, 2.08 to 3.74). The analysis conducted according to tertiles of serum GGT concentration yielded a similar pattern. In the final models, the RRs of GGT middle and top tertiles for death were 1.81 (95\% CI, 0.98 to 3.32 ) and 3.56 (95\% CI, 1.97 to 6.42 ), respectively (P for trend $<0.001$ ). Similarly, the RRs for 


\begin{tabular}{|c|c|c|c|c|c|}
\hline Subgroup & $\begin{array}{r}\text { No. of } \\
\text { Patients }\end{array}$ & & \multicolumn{2}{|c|}{ Relative Risk $(95 \% \mathrm{CI})$} & $P$ value \\
\hline Age & & & 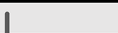 & & 0.615 \\
\hline$\leqslant 50$ years & 54 & & $\longmapsto \square$ & $1.69(1.14,2.50)$ & \\
\hline$>50$ years & 53 & & -1 & $1.50(1.04,2.16)$ & \\
\hline Sex & & & & & 0.523 \\
\hline Male & 72 & $\vdash$ & $=$ & $1.35(0.66,2.75)$ & \\
\hline Female & 35 & & $\vdash$ & $1.60(1.24,2.07)$ & \\
\hline HBV DNA & & & & & 0.252 \\
\hline$\leqslant 10 \sim 3$ copies $/ \mathrm{mL}$ & 22 & & $\mapsto \square$ & $1.97(1.46,2.66)$ & \\
\hline$>10 \sim 3$ copies $/ \mathrm{mL}$ & 85 & & $1-1$ & $1.55(1.15,2.10)$ & \\
\hline CA19-9 & & & & & 0.141 \\
\hline$\leqslant 37 \mathrm{u} / \mathrm{mL}$ & 49 & & $\longmapsto$ & $2.10(1.51,2.91)$ & \\
\hline$>37 \mathrm{u} / \mathrm{mL}$ & 58 & & $\vdash$ & $1.55(1.17,2.05)$ & \\
\hline Liver resection & & & & & 0.325 \\
\hline Local & 44 & & $\longmapsto$ & $1.96(1.40,2.76)$ & \\
\hline Anatomical & 63 & & $\vdash$ - & $1.59(1.18,2.14)$ & \\
\hline Adjuvant chemotherapy & & & & & $0.040 *$ \\
\hline Yes & 36 & $\vdash$ & $\longrightarrow$ & $0.64(0.22,1.89)$ & \\
\hline \multirow[t]{2}{*}{ No } & 71 & & $\vdash$ & $1.77(1.38,2.27)$ & \\
\hline & & 0 & 2 & 3 & \\
\hline
\end{tabular}

Figure 3 The correlation between serum GGT concentration and postoperative death events across prescribed subgroups. The negative correlation between serum GGT levels and OS did not differ significantly between groups stratified by age, sex, HBV DNA level, CA19-9 level and liver resection type (all $\mathrm{P}$ for interaction $>0.05$ ). However, this relationship became no longer significant if the patients were treated with postoperative adjuvant chemotherapy (P for interaction =0.04). GGT, gamma-glutamyl transpeptidase; OS, overall survival; HBV, hepatitis B virus.

Table 3 Comparison of perioperative complication rates among different GGT concentration groups based on the Clavien-Dindo classification

\begin{tabular}{|c|c|c|c|c|}
\hline Clavien-Dindo grade & High $(n=36)$ & Middle $(n=35)$ & Low $(n=36)$ & $\mathrm{P}$ \\
\hline II & 5 (13.9) & $4(11.4)$ & $3(8.3)$ & \\
\hline III & $2(5.6)$ & $2(5.7)$ & $2(5.6)$ & \\
\hline IV & $1(2.8)$ & $0(0.0)$ & $1(2.8)$ & \\
\hline
\end{tabular}

GGT, gamma-glutamyl transpeptidase.

recurrence of the corresponding tertiles were 1.70 (95\% CI, 0.93 to 3.10 ) and 3.27 (95\% CI, 1.77 to 6.06), respectively ( $\mathrm{P}$ for trend $=0.002$ ). Also as shown in Figure 2, patients with elevated GGT levels in the higher tertiles show worse prognosis. The median survival time for the high, middle and low GGT groups were 21, 40 and 56 months, respectively. The median recurrence free time for the high, middle and low GGT groups were 16, 31 and 51 months, respectively.

\section{Subgroup analysis}

The negative correlation between serum GGT concentration and OS was consistent across prescribed subgroups except adjuvant chemotherapy subgroups (Figure 3). There was no evidence of effect modification among young or old patients $(\mathrm{RR}=1.69$ vs. $1.50, \mathrm{P}$ for interaction $=0.615)$, among male or female patients $(\mathrm{RR}$ $=1.35$ vs. 1.60 , $\mathrm{P}$ for interaction $=0.523)$, among patients who had or did not have detectable serum HBV DNA (RR $=1.55$ vs. 1.97 , $\mathrm{P}$ for interaction $=0.252$ ), among patients with or without positive CA19-9 $(\mathrm{RR}=1.55$ vs. $2.10, \mathrm{P}$ for interaction $=0.141$ ) and among patients who received anatomical or local resection $(\mathrm{RR}=1.59$ vs. $1.96, \mathrm{P}$ for interaction $=0.325)$. However, compared with individuals who did not receive adjuvant chemotherapy, serum GGT 
concentration was no longer significantly correlated with OS among those who received adjuvant chemotherapy (RR $=1.77 v s .0 .64, \mathrm{P}$ for interaction $=0.040$ ).

\section{Postoperative complications}

Postoperative complications of 107 patients were described according to the Clavien-Dindo classification in Table 3. The overall incidence of complications is up to $73.8 \%$, but the vast majority of them were Clavien-Dindo grade I (55.1\%), including fever, nausea, vomiting and pain. A total of 18 patients (16.8\%) had grade II and III complications, including mild liver function abnormalities, pancreatitis, leukopenia, biliary system complications, and were cured by pharmacologic treatment or endoscopic retrograde cholangio-pancreatography. Both the high and low GGT groups had one patient who died from complications. Overall, there was no significant difference in the incidence of postoperative complications among these three groups.

\section{Discussion}

So far, the association between serum GGT and postoperative prognosis in patients with $\mathrm{HBV}$-associated ICC remains unclear. Our study showed an independent relationship between high serum GGT concentration and poor outcomes after adjustment of potential confounding factors in different multivariate models. Moreover, we found that this relationship became less significant with the implementation of adjuvant chemotherapy, suggesting that serum GGT levels may be an indicator for guiding adjuvant chemotherapy in patients with HBV-related ICC.

Previously, GGT has gained some attention as a postoperative prognostic biomarker for several tumors, such as ovarian cancer (15), endometrial cancer (16), and renal cell carcinoma (17). Especially in HCC, elevated serum GGT has been reported to be a biomarker of poor prognosis after hepatectomy (18), transcatheter arterial chemoembolization (19) and radiofrequency ablation (20). Results of our study extended these findings in HBVassociated ICC. In addition, it supported, to some extent, the early hypothesis that both HBV-associated HCC and ICC might be involved in mutual disease processes (21). Theoretically, one day clinicians might motivate subjects to change their lifestyles by limiting alcohol intake and other factors that can significantly increase GGT levels (22), thereby improving long-term postoperative outcomes.
More importantly, it is worth exploring whether shortening the follow-up interval for patients with high GGT levels can improve their prognoses.

In order to assess the robustness of the conclusion, we conducted a series of sensitivity analyses as follows. First, the results we obtained using this full sample set were not biased by the inclusion of various covariates. The stepwise adjustment of various different potential confounders did not strongly change the effect estimates. In addition, the correlation of serum GGT concentration with poor prognosis was verified by dividing the GGT into tertiles. In fact, most previous studies used GGT as a categorical variable rather than a continuous variable to assess its predictive effect (23-25). Therefore, the magnitude of the correlation could not be precisely determined. On the other hand, if we only treat GGT as a continuous variable, there is a possibility of ignoring the threshold effect which was observed previously in different study populations (26). In our study, whether the serum GGT concentration was classified as a continuous variable or a categorical variable, it remains significantly correlated with poor prognoses. Finally, we calculated the "E-value" to assess the bias caused by unmeasured or uncontrolled confounding factors. The E-value is the minimum strength of association, on the risk ratio scale, that an unmeasured confounder would need to have with both the treatment and outcome, conditional on the measured covariates, to explain a treatment-outcome association (27). Here, the E-value is $\mathrm{E}=2.83$ (95\% CI, 2.08 to 3.74 ) for the estimate. This indicates that a relatively strong confounding association would be needed to completely explain the observed causation of $\mathrm{RR}=1.72$.

With the advantage of interaction analyses, we also demonstrated that none of HBV levels, CA19-9 and liver resection types can substantially change the ability of serum GGT concentration on the forecast of patients' prognoses. Previously, it is indicated that these three variables might play important roles in tumor progression and metastasis. Of particular interest, the relationship between higher serum GGT concentration and worse postoperative outcomes became less significant with the implementation of adjuvant chemotherapy. While the use of adjuvant chemotherapy following resection of ICC has long been debated (28), our results provide important clinical evidence for choosing the suitable candidates. Further studies are needed to validate the guiding role of high serum GGT levels in the selection of adjuvant chemotherapy.

Given the epidemiologic nature of the observational 
study, it is still uncertain if GGT has a direct etiological role in tumor recurrence or may just be a marker of an underlying etiology. As reviewed by Kunutsor et al., GGT is positively and independently related to metabolic syndrome, diabetes, chronic kidney disease, dementia, and total mortality, thereby affecting postoperative survival (29). However, in this study, there was no significant correlation between high GGT concentrations and severe perioperative complications, and the prognosis was mainly affected by tumor recurrence. It is reasonable to propose some potential explanations for this phenomenon. First, early reports suggested the increased expression of GGT in actively proliferating pre-neoplastic foci in the liver (30) and GGTinduced damage could play an active role in the process by which cells of preneoplastic foci progress to malignancy (31). Therefore, these lesions may be an important source of postoperative tumor recurrence. Moreover, there is evidence that GGT is dysregulated in malignant cells. It can cause tumors to progress towards more aggressive phenotypes with worse prognoses by producing reactive oxygen species $(32,33)$. Consistent with these theories, patients with high GGT levels did show a higher recurrence rate in this study. Second, serum GGT level has also been characterized as a biomarker for oxidative stress, and was shown to correlate with inflammation in extracellular tissue microenvironment $(34,35)$. The essential impact of inflammatory microenvironment on all tumors, including ICC, is now generally accepted (36). Therefore, our subsequent research should try to figure out the mechanism underlying the findings in this article.

There are some potential limitations of this study merit to be mentioned in interpreting the study results. Firstly, although information on major risk factors was collected, some unmeasured variables might confound the relationship between GGT and postoperative outcomes, such as alcohol consumption and psychosocial status. Therefore, we provide the E-value to prove the causality in our study to be strong. Furthermore, it was impossible to correct the estimates for intra-individual variation in serum GGT levels, because our study only pertained to a single point in time. Finally, the recruitment model in this study allowed us to examine a fairly homogeneous and high-risk cohort. Therefore, the conclusion cannot be immediately applied to ICC populations with other etiologies and it is still essential to confirm our findings in different geographic regions and by independent randomized trials.

In summary, as a simple and convenient biological marker, serum GGT concentration is associated with an increased risk of postoperative death and tumor recurrence in patients with $\mathrm{HBV}$-associated ICC. However, this relationship became less significant with the implementation of adjuvant chemotherapy. Our findings, if confirmed, support that stratifying patients according to serum GGT concentration can be used for patient counselling, individualized postoperative adjuvant treatment and follow-up.

\section{Acknowledgments}

Funding: This work was supported by Program of Shanghai Municipal Commission of Health and Family Planning (20174Y0072), and the National Natural Science Foundation of China (81572301, 81802893, 81871924, $81502487,81572844)$.

\section{Footnote}

Reporting Checklist: The authors have completed the STROBE reporting checklist. Available at http://dx.doi. org/10.21037/atm-20-1616

Data Sharing Statement: Available at http://dx.doi. org/10.21037/atm-20-1616

Conflicts of Interest: All authors have completed the ICMJE uniform disclosure form (available at http://dx.doi. org/10.21037/atm-20-1616). The authors have no conflicts of interest to declare.

Ethical Statement: The authors are accountable for all aspects of the work in ensuring that questions related to the accuracy or integrity of any part of the work are appropriately investigated and resolved. This work was conducted in accordance with the Declaration of Helsinki (as revised in 2013) and was approved by ethics committee of Zhongshan Hospital (Y2017-250) and individual consent for this retrospective analysis was waived.

Open Access Statement: This is an Open Access article distributed in accordance with the Creative Commons Attribution-NonCommercial-NoDerivs 4.0 International License (CC BY-NC-ND 4.0), which permits the noncommercial replication and distribution of the article with the strict proviso that no changes or edits are made and the original work is properly cited (including links to both the formal publication through the relevant DOI and the license). 
See: https://creativecommons.org/licenses/by-nc-nd/4.0/.

\section{References}

1. Zhang $H$, Yang $T$, Wu $M$, et al. Intrahepatic cholangiocarcinoma: epidemiology, risk factors, diagnosis and surgical management. Cancer Lett 2016;379:198-205.

2. Razumilava N, Gores GJ. Cholangiocarcinoma. Lancet 2014;383:2168-79.

3. Choi SB, Kim KS, Choi JY, et al. The prognosis and survival outcome of intrahepatic cholangiocarcinoma following surgical resection: association of lymph node metastasis and lymph node dissection with survival. Ann Surg Oncol 2009;16:3048-56.

4. Tyson GL, El-Serag HB. Risk factors for cholangiocarcinoma. Hepatology 2011;54:173-84.

5. Kunutsor SK, Apekey TA, Van Hemelrijck M, et al. Gamma glutamyltransferase, alanine aminotransferase and risk of cancer: systematic review and meta-analysis. Int J Cancer 2015;136:1162-70.

6. Yin X, Zheng SS, Zhang BH, et al. Elevation of serum gamma-glutamyltransferase as a predictor of aggressive tumor behaviors and unfavorable prognosis in patients with intrahepatic cholangiocarcinoma: analysis of a large monocenter study. Eur J Gastroenterol Hepatol 2013;25:1408-14.

7. Zhang C, Wang H, Ning Z, et al. Serum liver enzymes serve as prognostic factors in patients with intrahepatic cholangiocarcinoma. Onco Targets Ther 2017;10:1441-9.

8. Huang J, Wang X, Zhu Y, et al. Specific prognostic factors in hepatitis $\mathrm{B}$ virus-related and non-hepatitis $\mathrm{B}$ virusrelated intrahepatic cholangiocarcinoma after macroscopic curative resection. J Surg Oncol 2019;119:40-6.

9. Fwu CW, Chien YC, You SL, et al. Hepatitis B virus infection and risk of intrahepatic cholangiocarcinoma and non-Hodgkin lymphoma: a cohort study of parous women in Taiwan. Hepatology 2011;53:1217-25.

10. Wang Q, Li J, Lei Z, et al. Prognosis of intrahepatic cholangiocarcinomas with $\mathrm{HBV}$ infection is better than those with hepatolithiasis after $\mathrm{r} 0$ liver resection: a propensity score matching analysis. Ann Surg Oncol 2017;24:1579-87.

11. Wakabayashi H, Hashimoto N, Okano K, et al. Clinicopathological comparison between intrahepatic cholangiocarcinoma arising in livers positive and negative for hepatitis B or C virus. Liver Int 2008;28:717-8.

12. Rizvi S, Gores GJ. Pathogenesis, diagnosis, and management of cholangiocarcinoma. Gastroenterology
2013;145:1215-29.

13. Wu ZF, Wu XY, Zhu N, et al. Prognosis after resection for hepatitis $B$ virus-associated intrahepatic cholangiocarcinoma. World J Gastroenterol 2015;21:935-43.

14. Dindo D, Demartines N, Clavien PA. Classification of surgical complications: a new proposal with evaluation in a cohort of 6336 patients and results of a survey. Ann Surg 2004;240:205-13.

15. Seebacher V, Polterauer S, Grimm C, et al. Prognostic significance of gamma-glutamyltransferase in patients with endometrial cancer: a multi-centre trial. Br J Cancer 2012;106:1551-5.

16. Grimm C, Hofstetter G, Aust S, et al. Association of gamma-glutamyltransferase with severity of disease at diagnosis and prognosis of ovarian cancer. Br J Cancer 2013;109:610-4.

17. Hofbauer SL, Stangl KI, de Martino M, et al. Pretherapeutic gamma-glutamyltransferase is an independent prognostic factor for patients with renal cell carcinoma. Br J Cancer 2014;111:1526-31.

18. Fu S, Guo Z, Li S, et al. Prognostic value of preoperative serum gamma-glutamyltranspeptidase in patients with hepatocellular carcinoma after hepatectomy. Tumour Biol 2016;37:3433-40.

19. Zhang JB, Chen Y, Zhang B, et al. Prognostic significance of serum gamma-glutamyl transferase in patients with intermediate hepatocellular carcinoma treated with transcatheter arterial chemoembolization. Eur J Gastroenterol Hepatol 2011;23:787-93.

20. Ma H, Zhang L, Tang B, et al. $\gamma$-Glutamyltranspeptidase is a prognostic marker of survival and recurrence in radiofrequency-ablation treatment of hepatocellular carcinoma. Ann Surg Oncol 2014;21:3084-9.

21. Lee CH, Chang CJ, Lin YJ, et al. Viral hepatitis-associated intrahepatic cholangiocarcinoma shares common disease processes with hepatocellular carcinoma. Br J Cancer 2009;100:1765-70.

22. Breitling LP, Raum E, Muller H, et al. Synergism between smoking and alcohol consumption with respect to serum gamma-glutamyltransferase. Hepatology 2009;49:802-8.

23. Hung TH, Tsai CC, Lin CC, et al. Is transarterial chemoembolization beneficial for patients with diffuse infiltrative hepatocellular carcinoma? Hepatol Int 2013;7:676-82.

24. Song P, Inagaki Y, Wang Z, et al. High levels of gammaglutamyl transferase and indocyanine green retention rate at $15 \mathrm{~min}$ as preoperative predictors of tumor recurrence in patients with hepatocellular carcinoma. Medicine 


\section{Page 10 of 10}

(Baltimore) 2015;94:e810.

25. Nishikawa H, Osaki Y, Iguchi E, et al. Radiofrequency ablation for hepatocellular carcinoma: the relationship between a new grading system for the ablative margin and clinical outcomes. J Gastroenterol 2013;48:951-65.

26. Breitling LP, Claessen H, Drath C, et al. Gammaglutamyltransferase, general and cause-specific mortality in 19,000 construction workers followed over 20 years. J Hepatol 2011;55:594-601.

27. VanderWeele TJ, Ding P. Sensitivity Analysis in Observational Research: Introducing the E-Value. Ann Intern Med 2017;167:268-74.

28. Ejaz A, Cloyd JM, Pawlik TM. Advances in the diagnosis and treatment of patients with intrahepatic cholangiocarcinoma. Ann Surg Oncol 2020;27:552-60.

29. Kunutsor SK. Gamma-glutamyltransferase-friend or foe within? Liver Int 2016;36:1723-34.

30. Roomi MW, Gaal K, Yuan QX, et al. Preneoplastic liver cell foci expansion induced by thioacetamide toxicity in drug-primed mice. Exp Mol Pathol 2006;81:8-14.

31. Stark AA, Russell JJ, Langenbach R, et al. Localization

Cite this article as: Zhang B, Liu S, Zhou B, Guo L, Li H, Yan J, Zhang W, Yu M, Chen Z, Xu Y, Xiao Y, Ye Q. High serum gamma-glutamyl transpeptidase concentration associates with poor postoperative prognosis of patients with hepatitis B virus-associated intrahepatic cholangiocarcinoma. Ann Transl Med 2021;9(1):17. doi: 10.21037/atm-20-1616
Zhang et al. High GGT levels associate with poor outcome of ICC

of oxidative damage by a glutathione-gamma-glutamyl transpeptidase system in preneoplastic lesions in sections of livers from carcinogen-treated rats. Carcinogenesis 1994;15:343-8.

32. Li T, Qin LX, Zhou J, et al. Staging, prognostic factors and adjuvant therapy of intrahepatic cholangiocarcinoma after curative resection. Liver Int 2014;34:953-60.

33. Hanigan MH, Frierson HF Jr, Swanson PE, et al. Altered expression of gamma-glutamyl transpeptidase in human tumors. Hum Pathol 1999;30:300-5.

34. Corti A, Franzini M, Paolicchi A, et al. Gammaglutamyltransferase of cancer cells at the crossroads of tumor progression, drug resistance and drug targeting. Anticancer Res 2010;30:1169-81.

35. Silva IS, Ferraz ML, Perez RM, et al. Role of gammaglutamyl transferase activity in patients with chronic hepatitis $\mathrm{C}$ virus infection. J Gastroenterol Hepatol 2004;19:314-8.

36. Elinav E, Nowarski R, Thaiss CA, et al. Inflammationinduced cancer: crosstalk between tumours, immune cells and microorganisms. Nat Rev Cancer 2013;13:759-71. 
Supplementary

Table S1 Univariate Cox regression analyses of risk factors related to OS and TTR in 107 patients

\begin{tabular}{|c|c|c|c|c|}
\hline \multirow{2}{*}{ Variables } & \multicolumn{2}{|l|}{ OS } & \multicolumn{2}{|l|}{ TTR } \\
\hline & Odds ratio $(95 \% \mathrm{Cl})$ & $P$ value & Odds ratio $(95 \% \mathrm{Cl})$ & $P$ value \\
\hline Age, years, $\leq 50$ vs. $>50$ & $0.72(0.46,1.12)$ & 0.146 & $0.69(0.45,1.07)$ & 0.098 \\
\hline TB, per SD & $0.98(0.79,1.21)$ & 0.846 & $1.01(0.83,1.23)$ & 0.916 \\
\hline Prothrombin time (s) & $1.16(0.95,1.43)$ & 0.155 & $1.18(0.97,1.44)$ & 0.093 \\
\hline Serum albumin, per SD & $0.78(0.62,0.99)$ & $0.039^{*}$ & $0.72(0.58,0.91)$ & $0.006^{*}$ \\
\hline Glutamic pyruvic transaminase, per SD & $0.98(0.80,1.20)$ & 0.830 & $1.00(0.82,1.21)$ & 0.988 \\
\hline GGT, per SD & $1.54(1.27,1.87)$ & $<0.001^{\star}$ & $1.53(1.26,1.86)$ & $<0.001^{\star}$ \\
\hline Tumor size $(\mathrm{cm})$ & $1.05(0.97,1.13)$ & 0.210 & $1.07(0.99,1.15)$ & 0.082 \\
\hline Sex (male vs. female) & $1.45(0.89,2.35)$ & 0.132 & $1.68(1.04,2.71)$ & $0.035^{\star}$ \\
\hline Cirrhosis (positive vs. negative) & $1.35(0.73,2.51)$ & 0.335 & $1.31(0.71,2.42)$ & 0.386 \\
\hline HBV DNA, copies/mL $\left(>10^{3}\right.$ vs. $\left.\leq 10^{3}\right)$ & $1.07(0.61,1.87)$ & 0.824 & $1.11(0.64,1.92)$ & 0.708 \\
\hline CA19-9, u/mL ( $\leq 37$ vs. $>37)$ & $1.02(0.65,1.59)$ & 0.945 & $1.44(0.92,2.24)$ & 0.108 \\
\hline AFP, ng/ml ( $\leq 20$ vs. $>20)$ & $1.30(0.77,2.18)$ & 0.328 & $1.34(0.80,2.26)$ & 0.262 \\
\hline Tumor number (multiple vs. solidary) & $1.39(0.60,3.20)$ & 0.438 & $1.15(0.50,2.65)$ & 0.738 \\
\hline Tumor location (single lobe vs. multiple lobes) & $2.41(1.38,4.20)$ & $0.002^{*}$ & $2.11(1.21,3.68)$ & $0.008^{*}$ \\
\hline Liver resection (anatomical vs. local) & $0.74(0.46,1.17)$ & 0.196 & $0.76(0.48,1.19)$ & 0.225 \\
\hline Tumor capsule (positive vs. negative) & $0.74(0.38,1.43)$ & 0.366 & $0.70(0.36,1.36)$ & 0.296 \\
\hline Micro thrombus invasion (yes vs. no) & $1.46(0.86,2.48)$ & 0.157 & $2.02(1.22,3.36)$ & $0.006^{*}$ \\
\hline Hilar lymph nodes (negative vs. positive) & $1.13(0.54,2.35)$ & 0.743 & $1.21(0.58,2.52)$ & 0.607 \\
\hline Histologic differentiation (well vs. poor) & $1.71(1.08,2.70)$ & $0.022^{*}$ & $1.71(1.09,2.68)$ & $0.019^{*}$ \\
\hline Adjuvant chemotherapy (yes vs. no) & $0.86(0.54,1.36)$ & 0.510 & $1.04(0.66,1.63)$ & 0.876 \\
\hline
\end{tabular}

*, Significant difference. OS, overall survival; TTR, time to recurrence; $\mathrm{Cl}$, confidence interval; TB, total bilirubin; SD, standard deviation; GGT, gamma-glutamyl transpeptidase; HBV, hepatitis B virus; CA19-9, carbohydrate antigen 19-9; AFP, alpha-fetoprotein. 


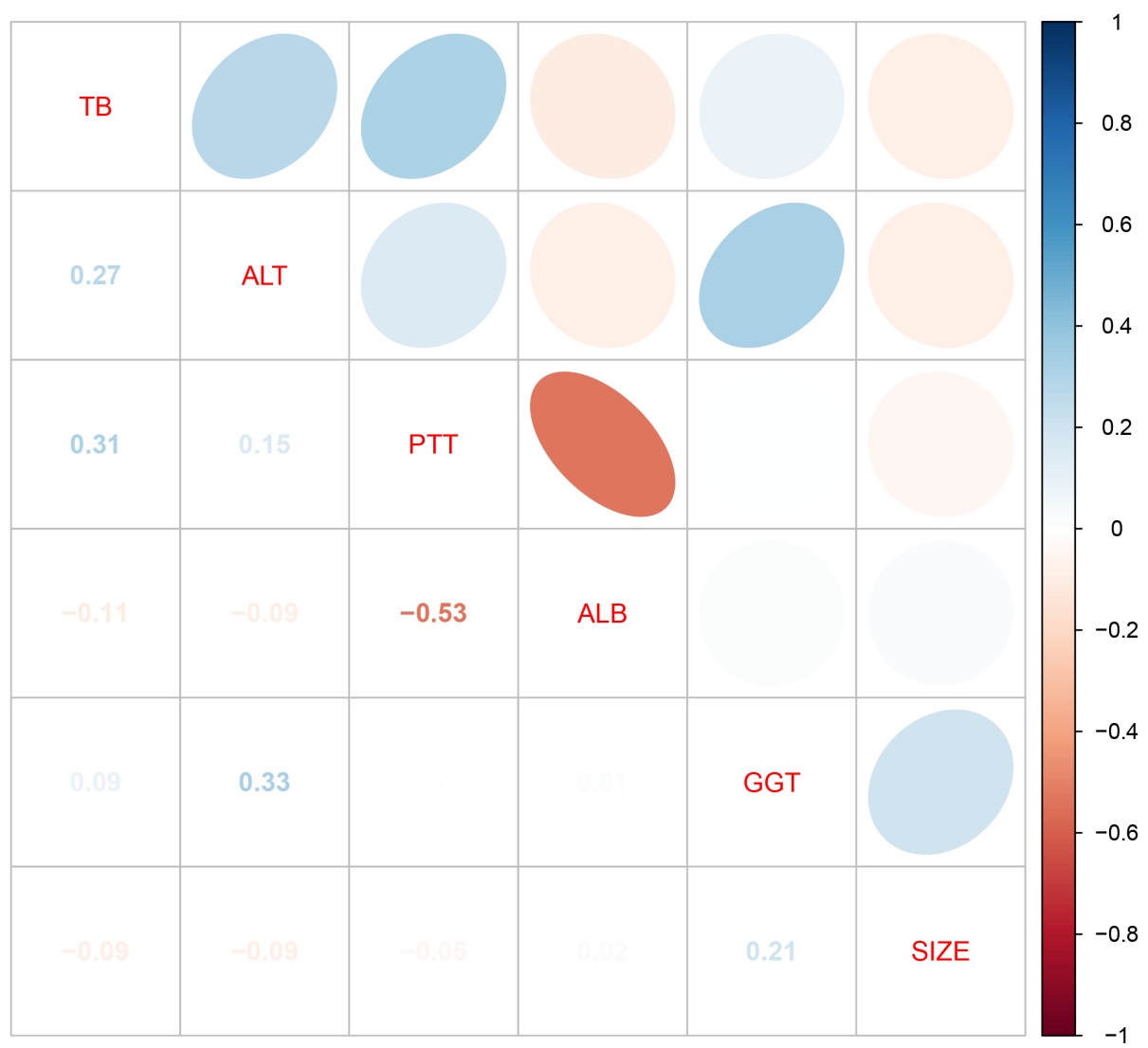

Figure S1 Factors potentially correlated with serum GGT concentration. Correlation test showed that all of the P value of correlation for each pair are $>0.05$. Even ALT which is the most relevant factor of GGT, has a correlation coefficient of only 0.33. GGT, gamma-glutamyl transpeptidase; ALT, alanine aminotransferase; TB, total bilirubin. 